\title{
VALUATED BUTLER GROUPS OF FINITE RANK
}

\author{
L. FUCHS ${ }^{\circ}$ and K. M. RANGASWAMY \\ Dedicated to the memory of B. H. Neumann
}

(Received 16 January 2004; revised 19 January 2005)

Communicated by E. A. O'Brien

\begin{abstract}
Valuated Butler groups of finite rank are investigated. The valuated $\boldsymbol{B}_{2}$-groups are both epic images and pure subgroups of completely decomposable valuated groups of finite rank (Theorem 3.1). However, there are fundamental changes in the theory of Butler groups when valuations are involved. We introduce valuated $B_{1}$-groups and show that they are valuated $B_{2}$-groups. Surprisingly, valuated $B_{2}$-groups of rank greater than 1 need not be valuated $B_{1}$-groups, unless they carry a special kind valuation, see Theorem 7.1. Theorem 6.5 gives a full characterization of valuated $B_{1}$-groups of finite rank, generalizing Bican's characterization of Butler groups.
\end{abstract}

2000 Mathematics subject classification: primary $20 \mathrm{~K} 15$.

Keywords and phrases: valuated group, Butler group, completely decomposable valuated group, valuated balanced subgroup, valuated $B_{1}$ - and $B_{2}$-groups.

\section{Introduction}

Butler groups as well as valuated groups are topics in the center of interest in abelian group theory. In this note, we wish to combine the two theories and initiate a general study of valuated Butler groups in the finite rank case.

A most important class of abelian groups, the so-called Butler groups, possesses several remarkable properties which have been investigated by a number of authors, see Arnold [1]. Finite rank Butler groups have been studied also by concentrating on free essential subgroups, furnished with the height valuation. Furthermore, in the classification of an important class of mixed abelian groups (called Warfield groups),

(C) 2006 Australian Mathematical Society $1446-7887 / 06 \$ A 2.00+0.00$ 
free abelian groups with ordinal valuation turned out to be crucial tools. Valuated free abelian groups have been studied by several authors (see, for example, [2, 3, 8]).

Our setting is more general: rather than free abelian groups we deal with genuine Butler groups equipped with valuations of ordinal values for every prime $p$; their theory cannot be reduced to the study of valuated free groups, because the valuations for the whole group cannot be recaptured from a subgroup. In this note, we restrict ourselves to groups of finite rank. Consequently, we will tacitly assume throughout that all torsion-free groups in this note are of finite rank.

Recall that a finite rank torsion-free group $B$ is called a Butler group if it satisfies one of the following equivalent conditions:

(1) (Butler [6]) $B$ is a pure subgroup of a completely decomposable group (by a completely decomposable group is meant a direct sum of torsion-free groups, each isomorphic to some subgroup of the rational group $\mathbb{Q}$ );

(2) (Butler [6]) $B$ is a surjective image of a finite rank completely decomposable group;

(3) (Bican [4]) The set $\Pi$ of prime numbers has a partition $\Pi=\Pi_{1} \cup \cdots \cup \Pi_{k}$ such that, for each $j$, the tensor product $B \otimes \mathbb{Z}_{\Pi_{j}}$ (the localization of $B$ at the set $\Pi_{j}$ of primes) is a completely decomposable group;

(4) (Bican and Salce [5]) Balanced extensions of torsion groups by $B$ are splitting, that is, $\operatorname{Bext}^{\mathrm{t}}(B, T)=0$ for all torsion groups $T$ ( $G$ is a balanced extension of $T$ by $B$ if the subgroups of $\mathbb{Q}$ have the projective property with respect to the exact sequence $0 \rightarrow T \rightarrow G \rightarrow B \rightarrow 0$ ).

Each of these properties are meaningful if the groups are furnished with a valuation. But are they still equivalent? This is the question which we want to answer in this note.

Our results will show that these four conditions are no longer equivalent in the setting of valuated groups, but partial equivalences and some implications are still valid. Theorem 3.1 asserts that (1) and (2) are equivalent for valuated Butler groups, that is, the classical equivalence theorem by Butler [6] carries over to the valuated situation (just as in the case of free valuated groups); we call these groups valuated $B_{2}$-groups. It is more challenging to verify the equivalence of (3) and (4) in the valuated case (Theorem 6.5) ((3) and (4) have not been considered as yet for free valuated groups); these groups will be called valuated $B_{1}$-groups - this terminology is in accordance with the standard terminology in the theory of Butler groups. It turns out that (3) [or (4)] implies (1) [and (2)], as is shown by Theorem 6.1. Somewhat surprisingly, the converse implication fails in general (see Examples 2 and 3). However, Butler groups equipped with the height valuation, and more generally, valuated $B_{2}$-groups with gap-free valuation, are still valuated $B_{1}$-groups (compare Corollary 7.2). In Theorem 7.1 we establish a necessary and sufficient condition for a valuated $B_{2}$-group to 
be a valuated $B_{1}$-group.

While our discussion of valuated $B_{2}$-groups makes use of methods already established in the theory of valuated groups, the study of valuated $B_{1}$-groups requires a new approach. The key seems to be the localization process; in fact, our results are based on a careful investigation of the local behavior of valuated $B_{1}$-groups. It will be clear that the fundamental difference between valuated $B_{1}$ - and $B_{2}$-groups lies in the local case: the former groups ought to be completely decomposable, but not the latter ones (Examples 2 and 3).

\section{Preliminaries}

We recall the definition of valuated groups. For a prime number $p$, by the $p$ valuation $v_{p}$ of an abelian group $G$ is meant a function from $G$ to the class of ordinals with the symbol $\infty$ adjoined (which is regarded to be larger than any ordinal) such that, for all $a, b \in G$,

(i) $v_{p}(a)=\infty$ if $a=0$;

(ii) $v_{p}(p a) \geq v_{p}(a)$, where strict inequality holds unless $v_{p}(a)=\infty$;

(iii) $v_{p}(n a)=v_{p}(a)$ whenever the integer $n$ is relatively prime to $p$;

(iv) $v_{p}(a+b) \geq \min \left(v_{p}(a), v_{p}(b)\right)$.

The valuation $v$ of the group $G$ is a collection of $p$-valuations $v_{p}$ of $G$, one for each prime $p$. Thus $v(a)$ for $a \in G$ is the sequence $v_{p}(a)$ of $p$-values of $a$ for $p=2,3,5, \ldots$.

The $p$-value of an element $a$ cannot be smaller than its $p$-height: $v_{p}(a) \geq h_{p}(a)$, where $h_{p}$ denotes the $p$-height. Thus, if $a \in G$ is an element of order $p^{k}$ (a prime power), then for all primes $q \neq p$ necessarily $v_{q}(a)=h_{q}(a)=\infty$. Also, if $a$ belongs to a $p$-divisible subgroup of $G$, then $v_{p}(a)=h_{p}(a)=\infty$.

In order to get more precise information about an element $a$ of a valuated group $G$, one also needs to know the values $v(n a)$ for each integer $n>0$. These values are known if we are given $v_{p}\left(p^{j} a\right)$ for each prime $p$ and for each non-negative integer $j$. Therefore we consider the value-matrix $V(a)$ of $a \in G$ which encodes all the needed value information about $a . V(a)$ is an $\omega \times \omega$ matrix whose $i, j$-entry is $v_{p}\left(p^{j} a\right)$ where $p$ is the $i$ th prime. It is easy to see that every $\omega \times \omega$-matrix with ordinal entries ( $\infty$ symbols are also admitted) is a value-matrix of some element in a suitable valuated group provided that the rows are strictly increasing (except when $\infty$ is reached). Value-matrices can be partially ordered pointwise.

A morphism $\phi$ between two valuated groups $G \rightarrow G^{\prime}$ is a group homomorphism that does not decrease values: $v_{p}(a) \leq v_{p}(\phi a)$ for all $a \in G$ and primes $p$. Valuated groups with these morphisms form a category $\mathscr{V}$. 
Two valuated groups $A, B$ are called isometric if there is a value-preserving isomorphism between them; we then write $A \approx B$ (and preserve the sign $\cong$ for pure group isomorphism). For example, two valuated free groups of rank 1 are isometric if and only if they can be generated by elements with the same value-matrix.

If $A$ is a subgroup of a valuated group $G$, then it is understood that the valuation in $A$ is the induced valuation from $G$, unless stated otherwise.

We assume familiarity with the basic notions in the theory of valuated groups, in particular, with the valuation of direct sums and of factor groups. By an exact sequence

$$
0 \rightarrow A \stackrel{\alpha}{\rightarrow} B \stackrel{\beta}{\rightarrow} C \rightarrow 0
$$

of valuated groups is meant an ordinary exact sequence such that $\alpha$ is a kernel and $\beta$ is a cokernel map, that is, $\alpha$ is an isometry between $A$ and $\operatorname{Im} \alpha$, and $\beta$ induces an isometry between $B / \operatorname{Im} \alpha$ and $C$.

It should be emphasized that the category $\mathscr{V}$ of valuated groups is not abelian, it is only pre-abelian in the sense that it is an additive category with kernels and cokernels. We shall use the fact, as pointed out by Richman and Walker in [9], that in such a category, and hence in $\mathscr{V}$, both pull-backs and push-outs exist, enjoying the usual universal properties.

We refer to Arnold [1] for results on finite rank Butler groups (without valuation); the basic facts will be used without explicit reference.

\section{Valuated $B_{2}$-groups}

By a completely decomposable valuated group is meant a group $F$ that is a direct sum of valuated torsion-free groups of rank 1 . In the finite rank case, we have $F \approx F_{1} \oplus \cdots \oplus F_{n}$ such that each $F_{i}$ is a rank 1 torsion-free valuated group and, for each prime $p, v_{p}\left(x_{1}+\cdots+x_{n}\right)=\min _{i} v_{p}\left(x_{i}\right)$ for $x_{i} \in F_{i}$.

Let $B$ be a finite rank torsion-free group which carries a valuation $v$. We call it a valuated $B_{2}$-group if there is a finite rank completely decomposable valuated group $F=F_{1} \oplus \cdots \oplus F_{n}$ (each $F_{i}$ is a valuated rank 1 torsion-free group) such that $B$ is isometric to the valuated factor group $F / H$ for some pure subgroup $H$ of $F$. Thus if $\phi: F \rightarrow B$ is the canonical map, then the elements $a_{i}=\phi x_{i},\left(x_{i} \in F_{i}\right)$, generate $B$ and valuation is given by $v(a)=\sup \min _{i} v\left(x_{i}\right)$, where the sup is taken for all possible representations of $a \in B$ as $a=\sum a_{i}, a_{i} \in \phi F_{i}$. Evidently, $B=\sum \phi F_{i}$ which is a valuated generation, that is, the valuation on $B$ is induced by the valuations on the $\phi F_{i}$ in the indicated way. From the definition of valuation in the image we can derive at once the formula

$$
B(p, \alpha)=\sum \phi F_{i}(p, \alpha)
$$


for each ordinal $\alpha$, where we have used the notation $B(p, \alpha)=\left\{b \in B \mid v_{p}(b) \geq \alpha\right\}$.

The class of valuated $B_{2}$-groups is evidently closed under epimorphisms. That it is also closed under the formation of pure subgroups will follow from the next theorem.

THEOREM 3.1. For a finite rank valuated torsion-free group $A$ the following are equivalent:

(i) A is an epic image of a completely decomposable valuated torsion-free group of finite rank;

(ii) $A$ is isometric to a pure subgroup of a completely decomposable valuated torsion-free group of finite rank.

Proof. (i) $\Rightarrow$ (ii) Let $A=\sum_{i=1}^{n} B_{i}$ with $n>1$ be a sum of rank 1 valuated torsion-free pure subgroups $B_{i}$ such that $n$ is minimal for $A$. Define $X_{i}=A / B_{i}$, $i=1, \ldots, n$, and consider the injection $\phi: A \rightarrow X_{1} \oplus \cdots \oplus X_{n}$ induced by the canonical valuated maps $A \rightarrow X_{i}$. The $X_{i}$ are valuated torsion-free groups of smaller rank with property (i), so if we show that this $\phi$ is value-preserving, then by induction we are done with the implication (i) $\Rightarrow$ (ii).

Assume to the contrary that there exist a prime $p$ and an element $a \in A$ such that $v_{p}(a)=\alpha$, but $v_{p}\left(a+B_{1}, \ldots, a+B_{n}\right)>\alpha$, that is, $v_{p}\left(a+B_{i}\right)>\alpha$ for every $i$. Pick $b_{i} \in B_{i}$ such that $v_{p}\left(a-b_{i}\right)>\alpha$. The last inequality means that there are $c_{i j} \in B_{j}$ with $v_{p}\left(c_{i j}\right)>\alpha$ satisfying $a-b_{i}=\sum_{j=1}^{n} c_{i j}$. Clearly, $v_{p}\left(b_{i}\right)=\alpha$, so there are integers $r_{i j}$, $s_{i j}$ such that $p \mid r_{i j}, p \nmid s_{i j}$ and $s_{i j} c_{i j}=r_{i j} b_{j}$ for all $i, j$. By modifying $r_{i j}$ if necessary, we may without loss of generality assume that $s_{i j}=s$ is the same for all $i, j$. This leads to the equations

$$
s a=\sum_{j=1}^{n}\left(\delta_{i j} s+r_{i j}\right) b_{j}, \quad i=1, \ldots, n,
$$

where det $\left\|\delta_{i j} s+r_{i j}\right\| \equiv s^{n}(\bmod p)$ with $\operatorname{gcd}(s, p)=1$. Hence Cramer's rule yields that each $b_{j}$ is linearly dependent on $a$, that is, $A$ is of rank 1 , a contradiction to $n>1$. Repeating the above arguments with height valuation instead of the given valuation $v$ (as is done in the theory of Butler groups), we get that the image of $\phi$ is pure in $X_{1} \oplus \cdots \oplus X_{n}$.

(ii) $\Rightarrow$ (i) Assume $A$ is a pure subgroup of the completely decomposable group $C=C_{1} \oplus \cdots \oplus C_{n}$, where the components $C_{i}$ are valuated torsion-free groups of rank 1 . The theory of Butler groups tells us that if $B_{1}, \ldots, B_{k}$ are the pure subgroups of $A$ with minimal supports in the direct sum, then they are of rank 1 and the canonical map $\psi: B=B_{1} \oplus \cdots \oplus B_{k} \rightarrow A$ is surjective. Thus it remains to check that the induced valuation on $B / \operatorname{Ker} \psi$ coincides with the valuation in $A$.

Fix a prime $p$, and let $a \in A$ with $v_{p}(a)=\alpha$. We show that $a$ has a preimage under $\psi$ with $p$-value $\alpha$. If $a$ belongs to some $B_{j}$, then this is obvious. Otherwise, 
there is a $b_{j} \in B_{j}$ for some $j$ such that $\operatorname{supp} b_{j} \subset \operatorname{supp} a$. Write $a=x_{1}+\cdots+x_{m}$, $b_{j}=y_{1}+\cdots+y_{\ell}, \ell<m$, with $x_{i}, y_{i} \in C_{i}$. As $B_{j}$ is pure in $A, b_{j} \in B_{j}$ can be chosen so as to have $v_{p}\left(x_{i}\right) \leq v_{p}\left(y_{i}\right)$ for all $i$ with $y_{i} \neq 0$, but for at least one index, say $i_{0}$, we have $y_{i_{0}}=x_{i_{0}}$. Then $a-b_{j} \in A$ has a smaller support than $a$, so inducting on the size of the support, we argue that $B$ contains an element $b^{\prime}$ such that $\psi\left(b^{\prime}\right)=a-b_{j}$ and $v_{p}\left(b^{\prime}\right)=v_{p}\left(a-b_{j}\right)$. By construction, $v_{p}\left(a-b_{j}\right) \geq \alpha$ and $v_{p}\left(b_{j}\right) \geq \alpha$, therefore

$$
v_{p}\left(b^{\prime}+b_{j}\right) \geq \min \left\{v_{p}\left(b^{\prime}\right), v_{p}\left(b_{j}\right)\right\}=\min \left(v_{p}\left(a-b_{j}\right), v_{p}\left(b_{j}\right) \geq \alpha=v_{p}(a) .\right.
$$

Since $\psi\left(b^{\prime}+b_{j}\right)=a$, the reverse inequality is obvious, and our claim follows.

The next example exhibits a finitely generated valuated group which is not a valuated $B_{2}$-group.

EXAMPLE 1. We define a valuated free group $F$ of rank two as follows. As a group, we set $F=\langle a\rangle \oplus\langle b\rangle$. Let $\alpha_{0}=0, \alpha_{1}, \alpha_{2}, \ldots, \alpha_{n}, \ldots$ be a strictly increasing sequence of ordinals - these will be the $p$-values of elements in $F$. The $p$-valuation $v_{p}$ in $F$ is given in terms of a transcendental $p$-adic unit $\pi$ whose $n$th partial sum will be denoted by $\pi_{n}$. For non-negative integers $k$ and $n$, set

$$
v_{p}\left(p^{k} a\right)=\alpha_{k}, \quad v_{p}\left(p^{k} b\right)=\alpha_{k}, \quad v_{p}\left(p^{k}\left(a+\pi_{n} b\right)\right)=\alpha_{k+n} .
$$

Furthermore, let $v_{q}(x)=\infty$ for all primes $q \neq p$ and all $x \in F$. It is straightforward to see that in this way every element of $F$ will have a well-defined value. $F$ cannot be embedded in a valuated free group as required by Theorem 3.1.

\section{Balanced subgroups}

Let $A$ be a subgroup of the group $G$ such that $G / A$ is torsion-free of rank 1 , and suppose that $G$ is equipped with a valuation $v$. We will say that $A$ is a (valuated) balanced subgroup in $G$ if there exist a valuated torsion-free group $X$ of rank 1 and an isometry $\phi: A \oplus X \rightarrow G$ with $\phi \mid A=\mathbf{1}_{A}$. In this case, every coset modulo $A$ in $G$ contains an element of maximal value-matrix.

If $A$ is pure and not of corank one in $G$, then $A$ is defined to be balanced in $G$ if it is balanced in every pure subgroup $C$ of $G$ that contains $A$ such that rank of $C / A$ is 1 . It is easily checked that balancedness is a transitive property.

An exact sequence $0 \rightarrow A \stackrel{\alpha}{\rightarrow} B \stackrel{\beta}{\rightarrow} C \rightarrow 0$ of valuated groups is said to be balanced-exact if $C$ is torsion-free and $\operatorname{Im} \alpha$ is a balanced subgroup in $B$.

It is clear from the definition that a balanced-exact sequence of torsion-free valuated groups is a balanced-exact sequence of ordinary groups (if we ignore the valuations).

Standard proofs apply to verify the following two lemmas. 
LEMMA 4.1. Let $0 \rightarrow A \rightarrow B \stackrel{\alpha}{\rightarrow} C \rightarrow 0$ be a valuated balanced-exact sequence where $C$ is torsion-free. Given a $\mathscr{V}$-map $\gamma: C^{\prime} \rightarrow C$, there is a pull-back diagram with balanced-exact top row:

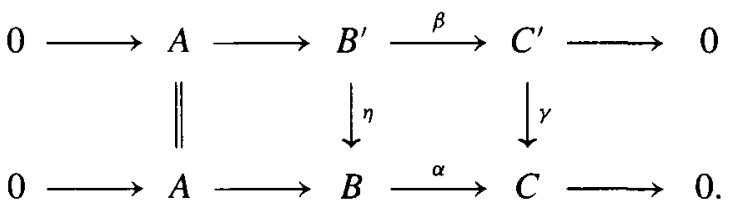

If $\gamma$ induces an isometry between $C^{\prime} / \operatorname{Ker} \gamma$ and $C$, then $\eta$ induces an isometry between $B^{\prime} / \operatorname{Ker} \eta$ and $B$.

A sort of dual to this lemma is the following result (we state it for the sake of completeness, though we will not need it).

LEMMA 4.2. Let $0 \rightarrow A \rightarrow B \stackrel{r}{\rightarrow} C \rightarrow 0$ be a valuated balanced-exact sequence where $C$ is torsion-free. For a $\mathscr{V}$-map $\alpha: A \rightarrow A^{\prime}$, there is a push-out diagram with balanced-exact bottom row:

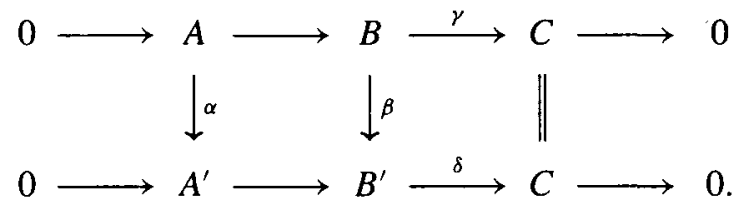

If $\alpha$ is an isometry of $A$ with $\operatorname{Im} \alpha$, then $\beta$ is an isometry of $B$ with $\operatorname{Im} \beta$.

\section{Valuated $B_{1}$-groups}

We now introduce the valuated analogue of what is called a $B_{1}$-group in the theory of Butler groups. (By the way, the results in this section are valid without restricting the ranks of the groups.) The definition is as follows.

A valuated torsion-free group $B$ will be called a valuated $B_{1}$-group if every valuated balanced-exact sequence $0 \rightarrow T \rightarrow G \rightarrow B \rightarrow 0$ of valuated groups is splitting for every valuated torsion group $T$. Equivalently, if $B$ has the projective property with respect to all valuated balanced-exact sequences of the mentioned type (that is, valuated extensions of torsion groups by torsion-free groups).

In view of the definition of balancedness, it is evident that all valuated rank 1 torsion-free groups are valuated $B_{1}$-groups. The same holds of course for their direct sums.

LEMMA 5.1. Completely decomposable valuated groups, as well as their summands are valuated $B_{1}$-groups. 
More generally, we can prove the following result that offers a sufficient condition for the $B_{1}$-property (compare Theorem 6.5).

If $G$ is a valuated group, then its localization at a prime $p$ is the group $G_{p}=G \otimes \mathbb{Z}_{p}$ (where $\mathbb{Z}_{p}$ denotes the localization of the ring $\mathbb{Z}$ at the prime $p$ ), whose valuation $w$ is given as follows: for an element $a / r \in G_{p}(a \in G, r \in \mathbb{Z}, \operatorname{gcd}(r, p)=1)$ and a prime $q, w_{q}(a / r)=v_{q}(a)$ or $\infty$ according as $q=p$ or $q \neq p . G_{p}$ is a $q$-divisible group, so it has to carry the height-valuation for every prime $q \neq p$. We say $G$ is $p$-local if it is identical with its own localization at $p$.

Similar definition of valuation applies if we localize at a set $\Pi^{\prime}$ of primes: the $p$-values are preserved for primes $p \in \Pi^{\prime}$, and are reset as $\infty$ otherwise.

THEOREM 5.2. A torsion-free valuated group $B$ is a valuated $B_{1}$-group if there is a partition $\Pi=\Pi_{1} \cup \cdots \cup \Pi_{k}$ of the set $\Pi$ of prime numbers such that for each $j$, $j=1, \ldots, k$, the tensor product $B \otimes \mathbb{Z}_{\Pi_{j}}$ is a completely decomposable valuated group.

ProOF. Let $\Pi=\Pi_{1} \cup \ldots \cup \Pi_{k}$ be a partition of primes such that $B \otimes \mathbb{Z}_{\Pi_{h}}$ is completely decomposable as a valuated group. If $0 \rightarrow T \rightarrow G \rightarrow B \rightarrow 0$ is a valuated balanced-exact sequence with $T$ torsion, then, for every $j=1, \ldots, k$, the sequence $0 \rightarrow T \otimes \mathbb{Z}_{\Pi_{j}} \rightarrow G \otimes \mathbb{Z}_{\Pi_{j}} \rightarrow B \otimes \mathbb{Z}_{\Pi_{j}} \rightarrow 0$ is also balanced-exact (under the induced valuations), so it splits. If $\phi_{j}: G \otimes \mathbb{Z}_{\Pi_{j}} \rightarrow T \otimes \mathbb{Z}_{\Pi_{j}}$ is a splitting map for the inclusion $T \otimes \mathbb{Z}_{\Pi} \rightarrow G \otimes \mathbb{Z}_{\Pi_{j}}$, then the composition

$$
G \rightarrow \bigoplus_{j=1}^{k}\left(G \otimes \mathbb{Z}_{n_{j}}\right) \stackrel{\phi}{\rightarrow} \bigoplus_{j=1}^{k}\left(T \otimes \mathbb{Z}_{\Pi_{j}}\right)=T
$$

is a valuated splitting map for the inclusion $T \rightarrow G$, where $\phi=\phi_{1}+\cdots+\phi_{k}$.

We proceed with the discussion by comparing valuated $B_{1}$-groups to $B_{1}$-groups. Recall that a $B_{1}$-group is defined as a (torsion-free) group $B$ satisfying

$$
\operatorname{Bext}^{1}(B, T)=0
$$

for all torsion groups $T$, that is, all balanced extensions of torsion groups by $B$ are splitting.

LEMMA 5.3. A $B_{1}$-group with its height valuation is a valuated $B_{1}$-group.

PROOF. Suppose $B$ is a torsion-free $B_{1}$-group equipped with its height valuation, and $G$ is a valuated extension of a valuated torsion group $T$ by $B$. We have a direct sum decomposition $G=T \oplus B^{\prime}$ as a group (that is, valuations are ignored for the time being) 
with $B^{\prime} \cong B$. First, observe that $B^{\prime}$ as a subgroup of $G$ must carry the height-valuation, because this is the minimal $p$-valuation. In order to see that this decomposition is a valuated decomposition, we have to show that $v_{p}\left(t, b^{\prime}\right) \leq \min \left(v_{p}(t), v_{p}\left(b^{\prime}\right)\right)$ for all $t \in T, b^{\prime} \in B^{\prime}$ (the reverse inequality being obvious). As the canonical homomorphism $G \rightarrow G / T$ maps $\left(t, b^{\prime}\right)$ upon $b \in B$ (the element corresponding to $b^{\prime} \in B^{\prime}$ ) and the valuation in $G / T$ must agree with the valuation in $B$, we clearly have $v_{p}\left(t, b^{\prime}\right) \leq v_{p}\left(b^{\prime}\right)$ for all $t \in T, b^{\prime} \in B^{\prime}$. In case $v_{p}(t)<v_{p}\left(b^{\prime}\right)$ we must have $v_{p}\left(t, b^{\prime}\right)=v_{p}(t)$, so also $v_{p}\left(t, b^{\prime}\right) \leq v_{p}(t)$. This completes the proof.

In view of Bican [4], the preceding lemma is a special case of our Theorem 6.5 below. A sort of converse is our next result.

THEOREM 5.4. Ignoring valuations, a valuated $B_{1}$-group is a $B_{1}$-group.

Proof. Assume $B$ is a valuated $B_{1}$-group and $T$ is any torsion group. Every extension $G$ of $T$ by the group $B$ (so far no valuations) may be viewed as a valuated extension. Indeed, furnish $T$ with the trivial valuation: $v_{p}(a)=\infty$ for all primes $p$ and for all $a \in T$, keep the valuation of $B$, and for $g \in G$ define $v(g)=v(g+T)$, the latter value being computed in $B$. This way $G$ becomes a valuated extension of $T$ by $B$. Moreover, if the extension $G$ is a balanced one, then it is a valuated balanced extension. Consequently, it splits. Hence, ignoring valuations, $B$ is indeed a $B_{1}$-group.

\section{Valuated $B_{1}$-groups of finite rank}

We now focus on finite rank valuated $B_{1}$-groups, and start with an improved version of Theorem 5.4. Though, as we shall see, the class of valuated $B_{2}$-groups is not identical with the class of valuated $B_{1}$-groups; we have an implication in one direction.

THEOREM 6.1. A finite rank valuated $B_{1}$-group is a valuated $B_{2}$-group.

Proof. By virtue of Theorem 5.4, a valuated $B_{1}$-group is a $B_{1}$-group. As is well known from the theory of Butler groups, $B_{1}$-groups are $B_{2}$-groups.

In order to prove the valuated part of the claim, suppose by way of contradiction that the valuated $B_{1}$-group $B$ is a Butler group of finite rank $r$, but not a valuated $B_{2}$-group, that is, it requires infinitely many pure subgroup generators to obtain its valuation, $B=\sum_{n<\omega} B_{n}$. Note that but finitely many of the $B_{n}$ may carry their height valuation (since such $B_{n}$ 's do not contribute anything to the valuation, it suffices to keep only those needed to generate $B$ as a $B_{2}$-group). Observe that if $v_{p}(b)>h_{p}(b)$ for some element $b \in B$, then also $v_{p}\left(p^{k} b\right)>h_{p}\left(p^{k} b\right)$ for all $k>0$. 
Let $A$ be an essential subgroup of $B$, and select subgroups $0 \neq K_{n} \subseteq A \cap B_{n}$ for $n<\omega$ such that there exist elements in $B_{n} \backslash K_{n}$ with higher than height-valuation whenever $B_{n}$ contains such elements. Let $\phi_{n}: B_{n} \rightarrow B_{n} / K_{n}=C_{n}$ denote the canonical map. Clearly, $A$ can be chosen such that $B$ contains a maximal independent set $x_{1}, \ldots, x_{r}$, none of which is contained in $A$; we may moreover assume without loss of generality that $x_{j} \in B_{j}, j=1, \ldots, r$.

Set $T=\bigoplus_{n<\omega} C_{n}$, a torsion group, and let $B^{\prime}$ be a group $\cong B$. Let $b_{n}^{\prime} \in B_{n}^{\prime}$ denote the element corresponding to $b_{n} \in B_{n}$ under a fixed isomorphism between $B$ and $B^{\prime}$. Define $G=T \oplus B^{\prime}$ with the valuation induced by:

(1) $T$ carries the height valuation;

(2) $B^{\prime}$ carries its height valuation;

(3) $v\left(\sum_{i=1}^{n} \phi_{i}\left(b_{i}\right), \sum_{i=1}^{n} b_{i}^{\prime}\right)=v\left(\sum_{i=1}^{n} b_{i}\right)$ for all $b_{n} \in B_{n}, n<\omega$.

In this way, $G$ becomes a valuated balanced-extension of $T$ by the valuated $B_{1}$ group $B$. By hypothesis, this is a valuated splitting extension, so there exists a subgroup $B^{*}$ in $G$ isometric to $B$, such that $G=T \oplus B^{*}$ (valuated direct sum). Then using * to denote elements in $B^{*}$ corresponding to those in $B$, note that changing $b_{n}^{\prime}$ to $b_{n}^{*}$ requires the addition of a torsion element, say $b_{n}^{*}=b_{n}^{\prime}+t_{n}$ with $t_{n} \in T$, where $t_{n} \neq 0$ for all $b_{n} \in B_{n} \backslash K_{n}$ for which $b_{n}$ is not valuated by its height. But once $x_{1}^{*}=b_{1}^{*}, \ldots, x_{r}^{*}=b_{r}^{*}$ have been fixed, all $b_{n}^{*}$ are fixed by linear dependence relations. Since we have infinitely many groups $C_{j}$, we can choose an index $j$ such that none of $t_{1}, \ldots, t_{r}$ has a coordinate in $C_{j}$. Then the linear combinations $b_{j}=\sum_{i=1}^{r} m_{i} x_{i}$ and $b_{j}^{*}=\sum_{i=1}^{r} m_{i} x_{i}^{*}, m_{i} \in \mathbb{Z}$, are contradictory, since they would imply $t_{j}=\sum_{i=1}^{r} m_{i} t_{i}$, which is clearly impossible, because $t_{j}$ must have a non-zero coordinate in $C_{j}$. Hence $B$ is indeed a valuated $B_{2}$-group.

Now that we know that finite rank valuated $B_{1}$-groups are valuated $B_{2}$-groups, our task is to single out those valuated $B_{2}$-groups that are also valuated $B_{1}$-groups.

We start with $p$-local valuated groups.

Before entering into the discussion of the $p$-local case, we recall from the theory of Butler groups that if $B$ is a Butler group, then its localization $B_{p}$ at any prime $p$ is a completely decomposable group: it is a direct sum of copies of $\mathbb{Z}_{p}$ and $\mathbb{Q}$. Thus we can write $B_{p}=A \oplus D$ where $A$ is a free $\mathbb{Z}_{p}$-module and $D$ is a divisible group. It should be emphasized that this is a valuated direct sum, since $D$ is trivially valuated, so $v_{q}(a, d)=v_{q}(a)$ for all $a \in A, d \in D$ and all primes $q$.

Central to our discussion is the fact stated in the following theorem.

THEOREM 6.2. A finite rank p-local valuated group $A$ is a valuated $B_{1}$-group if and only if it is a completely decomposable valuated group.

ProOF. Sufficiency is included in Lemma 5.1. To prove necessity, assume that $A$ 
is a valuated $B_{1}$-group. We know from Theorem 6.1 that it is a valuated $B_{2}$-group, so we can write $A=\sum_{i=1}^{n} B_{i}$ with rank 1 valuated pure groups $B_{i}$. As $A$ is $p$-local, each $B_{i}$ is isomorphic either to $\mathbb{Z}_{p}$ or to $\mathbb{Q}$.

Let $B_{1}, \ldots, B_{k}$ denote those $B_{i}$ 's whose $p$-valuation is not the height valuation, and $B_{k+1}, \ldots, B_{n}$ those which carry the height $p$-valuation (of course, the generator subgroups $B_{i}$ isomorphic to $\mathbb{Q}$ are in the second set). Without loss of generality we may assume that none of $B_{1}, \ldots, B_{k}$ can be omitted (that is, each of these contribute something to the valuation of $A$ ), and none of $B_{k+1}, \ldots, B_{n}$ can be omitted. It is important to keep in mind that the pure subgroups $B_{i}$ carry the same valuation as the one given in $A$.

Choose the integer $m$ such that each of $B_{1}, \ldots, B_{k}$ has elements not contained in $p^{m} A$ whose $p$-valuations exceed their $p$-heights (in particular, all the elements of ( $\left.\sum_{i=1}^{n} B_{i}\right) \cap p^{m} A$ have valuations exceeding their heights). Define $T_{j}, j=1, \ldots, k$ to be a cyclic $p$-group isomorphic to $B_{j} /\left(B_{j} \cap p^{m} A\right)$ with $\psi_{j}: B_{j} \rightarrow T_{j}$ as a fixed surjective map, and set $T=T_{1} \oplus \cdots \oplus T_{k}$. Define the valuated group $A^{\prime}$ to have the same underlying group as $A$, but its valuation is induced by $B_{1}^{\prime}, \ldots, B_{k}^{\prime}, B_{k+1}, \ldots, B_{n}$, where $B_{j}^{\prime}$ is isomorphic to $B_{j}$, with no change in the valuations of elements in $p^{m} A$, but for elements not in $p^{m} A$ the $p$-valuation is just the height valuation. Define $G=T \oplus A^{\prime}$ (pure group-theoretically), and furnish it with the valuation induced by:

(a) the height valuation of $T$;

(b) the indicated valuation of $A^{\prime}$;

(c) $v_{p}\left(\sum_{i=1}^{n} \psi_{i}\left(b_{i}\right), \sum_{i=1}^{n} b_{i}^{\prime}\right)=v_{p}\left(\sum_{i=1}^{n} b_{i}\right)$ for all $b_{j}^{\prime} \in B_{j}^{\prime}, j \leq n$,

where $b_{j} \in B_{j}$ and $b_{j}^{\prime} \in B_{j}^{\prime}$ denote corresponding elements under a fixed group isomorphism $A \cong A^{\prime}$. It is readily checked that $G$ is a valuated balanced-extension of $T$ by $A$. As $A$ was supposed to be a valuated $B_{1}$-group, there exists a valuated complement $A^{*} \approx A$ to $T$ in $G, G=T \oplus A^{*}$ (valuated direct sum). Evidently, $A^{*}=\sum_{i=1}^{n} B_{i}^{*}$ with $B_{i}^{*} \approx B_{i}$ for each $i$, and none of the $B_{i}^{*}$ can be omitted. There is a group isomorphism $\sigma_{i}: B_{i}^{\prime} \rightarrow B_{i}^{*}$ (the restriction of $A^{\prime} \rightarrow A$ ) such that corresponding elements are mapped upon the same element of $B_{i}$ by the canonical map $G \rightarrow A$. For $j=1, \ldots, k$ we write $\sigma_{j}\left(b_{j}^{\prime}\right)-b_{j}^{\prime}=\psi_{j}\left(b_{j}\right)+t_{j}$, where $t_{j}$ is a torsion element such that $h_{p}\left(t_{j}\right) \geq v_{p}\left(\sigma_{j}\left(b_{j}^{\prime}\right)\right)=v_{p}\left(b_{j}\right)>h_{p}\left(\psi_{j}\left(b_{j}\right)\right)$; the last inequality holds whenever $b_{j}$ is not height-valued (compare $p$-values, taking (c) into consideration). By way of contradiction, suppose there is a dependence relation between $B_{1}^{\prime}, \ldots, B_{k}^{\prime}$, say $\sum_{j=1}^{k} b_{j}^{\prime}=0$, where $b_{j}^{\prime} \in B_{j}^{\prime}$. We may assume that also $b_{j} \notin p^{m} A$ and $v_{p}\left(b_{j}\right)>$ $h_{p}\left(b_{j}\right)$ for all $j$, since if necessary we can increase $m$. Then $t_{j} \neq 0$, and also $\sum_{j=1}^{k} \sigma_{j}\left(b_{j}^{\prime}\right)=0$, whence

$$
\sum_{j=1}^{k} \psi_{j}\left(b_{j}\right)=-\sum_{j=1}^{k} t_{j}
$$


follows. Here the terms on the left are independent and carry their height valuations, so the $p$-value on the left is the minimum of the $p$-heights of the $\psi_{j}\left(b_{j}\right)$, which is certainly less than any $v_{p}\left(t_{j}\right)$. We reached a contradiction. Consequently, $C=B_{1} \oplus \cdots \oplus B_{k}$ must be a valuated direct sum.

Any subgroup with height valuation ought to be independent of $C$, because every non-zero element of $p^{m} C$ has valuation higher than its height. Thus, if we set $C^{*}=B_{k+1}+\cdots+B_{n}$, then we have a valuated direct sum decomposition $A=C \oplus C^{*}$. Here the reduced part of $C^{*}$ is a free $\mathbb{Z}_{p}$-module with height valuation, so $C^{*}$ is a completely decomposable valuated group. This completes the proof.

We wish to record a consequence of the last proof which will be needed later on.

COROLLARY 6.3. Let $A=\sum_{i=1}^{n} B_{i}$ be a p-local valuated $B_{1}$-group with rank 1 valuated pure subgroups $B_{i}$. There is a subset $\left\{B_{1}, \ldots, B_{\ell}\right\}$ of the set $\left\{B_{1}, \ldots, B_{n}\right\}$ of generator subgroups such that $A=B_{1} \oplus \cdots \oplus B_{\ell}$ is a valuated direct sum.

ProOF. It remains to show that $C^{*}$ is a direct sum of some of the $B_{i}$. But this follows at once from Nakamaya's lemma, noting that $\mathbb{Z}_{p}$ is a local domain.

The next lemma will be most helpful in reducing the global case to the local one.

LEMMA 6.4. If $B$ is a valuated $B_{1}$-group, then so is its localization $B_{p}$ for every prime $p$.

PROOF. In accordance with the above notation, we write $B_{p}=A \oplus D$, where $A$ is a free $\mathbb{Z}_{p}$-module and $D$ is a divisible group. As a divisible group, $D$ is a trivially valuated completely decomposable group, so $D$ is a valuated $B_{1}$-group. It remains to show that $A$ is a valuated $B_{1}$-group. Since $A$ is a $q$-divisible $B_{1}$-group for all primes $q \neq p$, only the balanced extensions of valuated $p$-groups by $A$ need to be considered.

Let $G$ be a valuated balanced extension of a valuated $p$-group $T$ by $B_{p}$; the group $B_{p}$ is now assumed to be reduced (that is, has no divisible subgroups $\neq 0$ ). Let $\sigma$ denote the natural injection $B \rightarrow B_{p}$. This is a $\mathscr{V}$-map, so we can form the valuated pull-back diagram

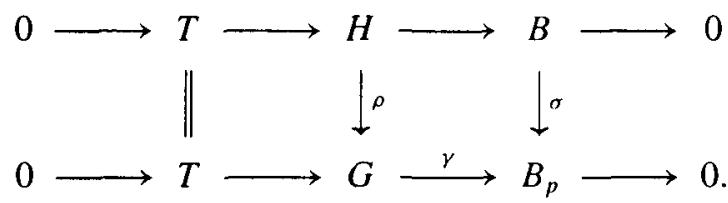

By Lemma 4.1, the top row is balanced-exact in the valuated sense, so in view of the hypothesis on $B$ it splits. Hence there is a valuated map $\beta: B \rightarrow G$ such that $\gamma \beta=\sigma$. Consider the exact sequence

$$
\operatorname{Hom}\left(B_{p}, G\right) \rightarrow \operatorname{Hom}(B, G) \rightarrow \operatorname{Ext}\left(B_{p} / B, G\right)
$$


of abelian groups. Here $B_{p} / B$ is a divisible torsion group whose $p$-component is 0 , while $G$ is $q$-divisible for every prime $q \neq p$. Observing that every extension of a $q$ divisible group by a $q$-group splits (see [7, page 223]), it follows that $\operatorname{Ext}\left(B_{p} / B, G\right)=$ 0 . Hence every group homomorphism from $B$ to $G$ extends to one from $B_{p}$ to $G$. We conclude that $\beta$ extends to a map $\alpha: B_{p} \rightarrow G$ which has to be a valuated map, since $\beta$ was one (recall the definition of valuations in localizations). Thus $\operatorname{Im} \alpha \approx B_{p}$.

Manifestly, $T \cap \operatorname{Im} \beta=0$ implies $T \cap \operatorname{Im} \alpha=0$, thus $G=T \oplus \operatorname{Im} \alpha$ is a direct sum. To see that this is a valuated direct sum, note that for the $p$-valuation this holds, because $\beta$ and hence $\alpha$ preserves $p$-values, while for $q$-values (for primes $q \neq p$ ) this is evident, since they are all $\infty$.

It remains to characterize the valuated $B_{1}$-groups in the global case. This is our main result for valuated $B_{1}$-groups.

THEOREM 6.5. A valuated torsion-free group $B$ of finite rank is a valuated $B_{1}$-group if and only if there exists a partition $\Pi=\Pi_{1} \cup \ldots \cup \Pi_{k}$ of the set $\Pi$ of prime numbers such that the tensor product $B \otimes \mathbb{Z}_{\Pi}, j=1, \ldots, k$, is a completely decomposable valuated group.

PROOF. Theorem 5.2 takes care of the proof of sufficiency.

Turning our attention to the proof of necessity, suppose that $B=\sum_{i=1}^{n} B_{i}$ is a valuated $B_{1}$-group of rank $r$. From Lemma 6.4 we know that the localizations $B_{p}$ of $B$ at primes $p$ are likewise $B_{1}$-groups, while Corollary 6.3 shows that $B_{p}$ is the valuated direct sum of some of the localized generators $B_{i}$; of course, of exactly $r$ of them. For every prime $p$, choose an $r$-element subset $X$ of the set $\left\{B_{1}, \ldots, B_{n}\right\}$ of the generating groups such that the localization $B_{p}$ is the valuated direct sum of the localized $B_{i}$ in $X$, and define $\Pi_{X}$ as the set of all primes $p$ to which $X$ is assigned. Let $\Pi_{1}, \ldots, \Pi_{k}$ be the list of the non-empty sets $\Pi_{X}$. Clearly, $\Pi=\Pi_{1} \cup \cdots \cup \Pi_{k}$ is a partition of the set $\Pi$ of primes. From the theory of torsion-free groups we know that if we consider, as we may, localizations as subgroups in the divisible hull, then we have $B=\bigcap_{j=1}^{k}\left(B \otimes \mathbb{Z}_{\Pi_{j}}\right)$ (intersection in the divisible hull of $B$ ). Since the $p$-valuation of the elements of $B$ in $B \otimes \mathbb{Z}_{\Pi_{j}}$ is either the same as in $B$ or is $\infty$ according to whether $p$ belongs to the class of partition corresponding to $\Pi_{j}$ or not, this intersection yields the correct valuations of the elements of $B\left(v_{p}(a)\right.$ in the intersection is defined as the infimum of the $p$-values of $a$ in the components). That $B \otimes \mathbb{Z}_{\Pi_{j}}$ is a completely decomposable valuated group is evident from the definition.

\section{When valuated $B_{2}$-groups are valuated $B_{1}$-groups}

Theorem 6.1 states that all valuated $B_{1}$-groups of finite rank are valuated $B_{2}$-groups. We now exhibit two examples to show that the converse implication fails in general. 
(For those familiar with the theory of Butler groups of arbitrary cardinality, this fact should be somewhat surprising, since the converse implication is expected to hold.)

Our examples are $p$-local valuated $B_{2}$-groups that fail to be valuated $B_{1}$-groups. Both examples are given as epimorphic images of completely decomposable valuated groups of finite rank.

EXAMPLE 2. We make the free group $F=\langle a\rangle \oplus\langle b\rangle$ with basis $a, b$ into a valuated $p$-local group as follows ( $p$ is an arbitrary, but fixed prime). We assign the following $p$-values to $p^{k} a, p^{k} b, p^{k}(a+b)$, for $k=0,1, \ldots: 0,2,5,6,8, \ldots ; 0,3,4,6,9, \ldots$; and $1,2,4,7,8, \ldots$, respectively (the increases are periodically $2,3,1 ; 3,1,2 ;$ and $1,2,3)$. This is a valuated $B_{2}$-group, but not a valuated $B_{1}$-group, since the subgroups $\langle a\rangle,\langle b\rangle,\langle a+b\rangle$ are dependent, and $F$ is not a completely decomposable valuated group (see Theorem 6.2). This $F$ is an epic image of the valuated free group $F^{\prime}=$ $\langle a\rangle \oplus\langle b\rangle \oplus\langle c\rangle$ where the summands are valuated in the same way as the subgroups $\langle a\rangle,\langle b\rangle,\langle a+b\rangle$ are in $F$.

In the second example, the generating subgroups are almost isometric.

EXAMPLE 3. Let $p$ be a prime and $A$ the epic image of the direct sum $B=\langle x\rangle \oplus$ $\langle y\rangle \oplus\langle z\rangle$ modulo the pure subgroup $\langle x-y+z\rangle$ where the $p$-values of $p^{k} x, p^{k} y, p^{k} z$, for $k=0,1, \ldots$, are $0,3,4,6,7,8, \ldots ; 0,2,5,6,7,8, \ldots ;$ and $1,2,4,6,7,8, \ldots$, respectively (thus, for example, $v_{p}\left(p^{k} x\right)=k+3$ if $k \geq 3$ ), while the $q$-values at other primes $q$ are $\infty$. If $x^{*}$ and $z^{*}$ denote the images of $x$ and $z$ in $A$, then it is easy to verify that $x^{*}$ and $x$ (similarly, $z^{*}$ and $z$ ) have the same value-matrix. In order to see that $A=\langle a\rangle \oplus\langle b\rangle$ (valuated) is impossible for any choice of $a, b \in A$, note first that if this was a valuated decomposition, then one of $a, b$ had $p$-value 1 and the other had $p$-value 0 , say $v_{p}(a)=1$ and $v_{p}(b)=0$. It can then be seen that, for $k=0,1,2$, $v_{p}\left(p^{k} a\right)=v_{p}\left(p^{k} z\right)$ and $v_{p}\left(p^{k} b\right)=v_{p}\left(p^{k} x\right)$. Furthermore, if $x^{*}=k a+\ell b$ and $z^{*}=m a+n b(k, \ell, m, n \in \mathbb{Z})$, then we have $p \mid k$ and $\operatorname{gcd}(p, \ell)=1=\operatorname{gcd}(p, m)$. This yields that $p^{2} x^{*}=p^{2} z^{*}=(k+m) p^{2} a+(\ell+n) p^{2} b$ has $p$-value 4. But the element $p^{2} x^{*}+p^{2} z^{*} \in A$ is the image of $p^{2} y \in B$, so its $p$-value has to be $\geq 5$.

It is an obvious question: under what conditions is a valuated $B_{2}$-group a valuated $B_{1}$-group? We answer this question satisfactorily in the next theorem (all groups are of finite rank).

THEOREM 7.1. A valuated $B_{2}$-group $B$ of finite rank is a valuated $B_{1}$-group if and only if all of its localizations $B_{p}$ at primes $p$ are completely decomposable valuated groups.

Proof. By virtue of Theorem 6.5, the condition is necessary. 
To prove sufficiency, write $B=\sum_{i=1}^{n} B_{i}$ with rank 1 pure subgroups $B_{i}$. Observe that the hypothesis implies that every localization $B_{p}$ is a local $B_{1}$-group. Hence from Corollary 6.3 we deduce that for every prime $p$, there is a subset of $\left\{B_{1}, \ldots, B_{n}\right\}$ such that $B_{p}$ is the valuated direct sum of the localizations at $p$ of the groups in this set. We now argue as in the proofs of Lemma 6.4 and Theorem 6.2 that there is a partition of the set of primes as required by this theorem. Consequently, $B$ is a valuated $B_{1}$-group.

By making use of this criterion, we can generalize Lemma 5.3 to valuated $B_{2}$-groups whose valuations are gap-free in the sense that

$$
v_{p}(p a)=v_{p}(a)+1
$$

for all elements $a$ and all primes $p$ provided that $v_{p}(a) \neq \infty$.

COROLLARY 7.2. A valuated $B_{2}$-group of finite rank with gap-free valuation is a valuated $B_{1}$-group.

PROOF. By Theorem 7.1, it is enough to show that a $p$-local valuated Butler group $B$ with gap-free valuation is completely decomposable. As the divisible part $D$ of $B$ is a completely decomposable summand with trivial valuation and $F=B / D$ is a finitely generated free $\mathbb{Z}_{p}$-module, we may restrict the proof to the case $B=F$.

Thus assume $B=\sum_{i=1}^{n} B_{i}$ with $B_{i}=\mathbb{Z}_{p} b_{i}$ for some $b_{i} \in B_{i}$. Here the $b_{i}$ are of $p$-height 0 ; their values $v_{p}\left(b_{i}\right)=\alpha_{i}$ are assumed to satisfy $\alpha_{1} \geq \alpha_{2} \geq \cdots \geq \alpha_{n}$. Choose a maximal independent set $\left\{b_{1}, \ldots, b_{j}\right\}$ of maximal value $\alpha_{1}$, then extend this set to a maximal independent set $\left\{b_{1}, \ldots, b_{j}, \ldots, b_{h}\right\}$ with elements whose $p$ values are maximal among the non-maximal $\alpha_{i}$, and keep going. At the end of this process we obtain an independent set $\left\{b_{1}, \ldots, b_{j}, \ldots, b_{h}, \ldots, b_{k}\right\}$ that generates the free $\mathbb{Z}_{p}$-module $B$, since the $b_{i}$ generate $B / p B$ (argue with Nakayama's lemma), so $B=\bigoplus_{i=1}^{k} \mathbb{Z}_{p} b_{i}$. This is easily seen to be a valuated direct sum, due to the construction and the gap-free hypothesis. Thus $B$ is a completely decomposable valuated group.

In view of this corollary, we can claim that for valuated torsion-free groups of finite rank with gap-free valuation, conditions (1)-(4) listed in the introduction and rephrased for the valuated case are equivalent.

It is natural to raise the question whether or not pure subgroups and epimorphic images of finite rank $B_{1}$-groups are again of the same kind. The answer is in the negative: Example 3 is a counterexample. Indeed, the group $A$ in this example is an epic image of a completely decomposable valuated $p$-local group $B$, but it is not completely decomposable, so by Theorem 6.2 it is not a valuated $B_{1}$-group. By Theorem 3.1 , the group $A$ is also a pure subgroup of a completely decomposable 
valuated group. Thus the class of valuated $B_{1}$-groups of finite rank is not closed either under taking epic images or under pure subgroups (but it is obviously closed under the formation of valuated direct summands).

\section{References}

[1] D. M. Arnold, Abelian groups and representations of finite partially ordered sets, CMS Books in Mathematics/Ouvrages de Mathématiques de la SMC, 2 (Springer, New York, 2000).

[2] D. M. Arnold, R. Hunter and F. Richman, 'Direct sums of cyclic valuated groups', Sympos. Math. 23 (1979), 77-84.

[3] D. M. Arnold and F. Richman, 'Subgroups of finite direct sums of valuated cyclic groups', J. Algebra 114 (1998), 1-15.

[4] L. Bican, 'Purely finitely generated abelian groups', Comment. Math. Univ. Carolin. 21 (1980), 209-218.

[5] L. Bican and L. Salce, 'Butler groups of infinite rank', in: Abelian group theory, Lecture Notes Math. 1006 (Springer, 1983) pp. 171-189.

[6] M. Butler, 'A class of torsion-free abelian groups of finite rank', Proc. London Math. Soc. 15 (1965), 680-698.

[7] L. Fuchs, Infinite abelian groups, vol. 1 and 2 (Academic Press, New York, 1970 and 1973).

[8] L. Fuchs and G. Viljoen, 'Valuated Butler groups of special type', Czechoslovak Math. J. 49 (1999), 507-516.

[9] F. Richman and E. Walker, 'Ext in pre-abelian categories', Pacific J. Math. 71 (1977), 521-535.

Department of Mathematics

Tulane University

New Orleans

Louisiana 70118

USA

e-mail: fuchs@tulane.edu
Department of Mathematics

University of Colorado

Colorado Springs

Colorado 80933

USA

e-mail: ranga@math.uccs.edu 